Pierre-Alain Duc, Jonathan Braine and Elias Brinks, eds.

\title{
Superwind and Chain Galaxy Formation at High Redshift
}

\author{
Yasuhiro Shioya and Yoshiaki Taniguchi \\ Astronomical Institute, Tohoku University, Sendai, 980-8578, Japan
}

\begin{abstract}
We propose a possible new model for the formation of chain galaxies at high redshift.
\end{abstract}

\section{Introduction}

Recent high-resolution optical imaging studies of some deep fields have revealed a new population of galaxies at high redshift: chain galaxies (Cowie, Hu \& Songaila 1995; van den Bergh et al. 1996). The chain galaxies tend to be very straight in morphology; their average major-to-minor axial ratio is 5 . Cowie et al. (1995) proposed that the chain galaxies comprise a new population of forming galaxies at high redshift because there is no local counterpart. We propose a possible new model for the formation of chain galaxies at high redshift (Taniguchi \& Shioya 2001).

\section{Model and Discussion}

Our model is summarized as follows. (1) Successive merging of subgalactic gas clumps results in the formation of a galaxy with a mass of $10^{11}-10^{12} M_{\odot}$ at redshift $z \sim 5$. (2) Subsequently, supernova explosions occur inside the galaxy and then blow out as a galactic wind (or a superwind). This wind expands into the intergalactic space and then causes a large-scale shell with a radius of several hundred kiloparsecs. (3) Since the shell can be regarded as a gaseous sheet, filament-like gravitational instability is expected to occur. (4) Further gravitational instability occurs in each filament, leading to intense star formation along the filament. This is the chain galaxy phase. (5) The filament collapses gravitationally into one spheroidal system like an elliptical galaxy within one dynamical timescale of the filament $\left(\sim 10^{8} \mathrm{yr}\right)$. We also discuss that shocked shells driven by superwinds may be responsible for some Lyman limit systems and damped Ly $\alpha$ systems. A schematic illustration is shown in Figure 1.

\section{References}

Cowie, L. L., Hu, E. M., \& Songaila, A. 1995, AJ, 110, 1576

Taniguchi, Y. \& Shioya, Y. 2001, ApJ, 547, 146

van den Bergh, S., et al. 1996, AJ, 112, 359 

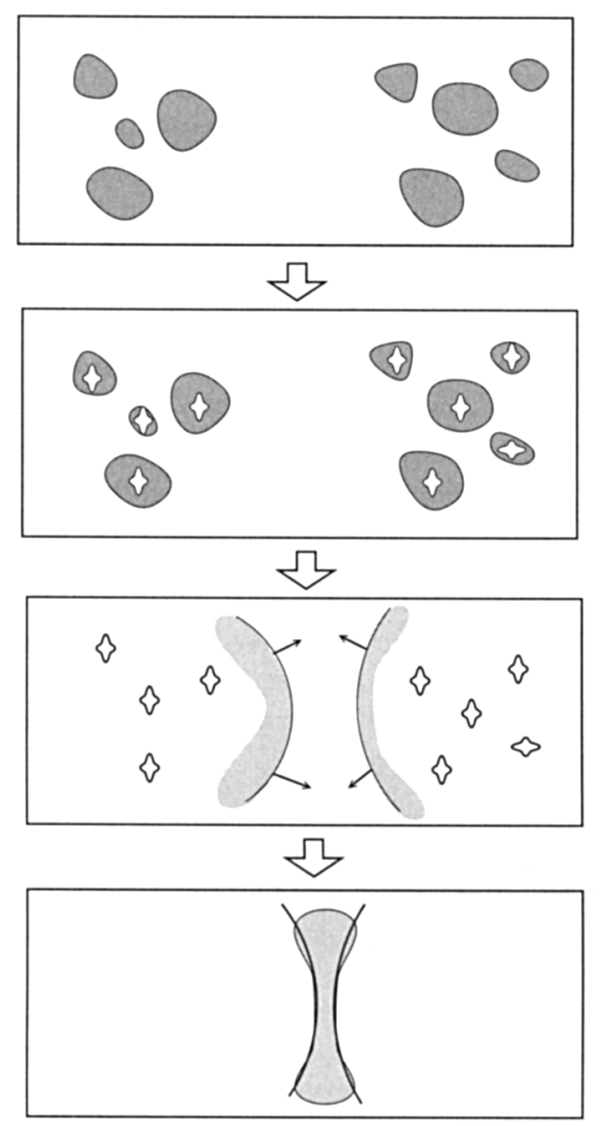

Overdense regions (or proto clusters of galaxies)

Starburst in the protogalaxies

Overlap of galactic winds and the formation of cosmic blast wave

Interaction of cosmic blast waves and the formation of cosmic slab
Filament-like instability in the slab
Formation of chain galaxies and evolution to elliptical-like galaxies

Figure 1. Schematic illustration of the proposed formation mechanism of chain galaxies. 